\title{
Systemic alendronate prevents resorption of necrotic bone during revascularization. A bone chamber study in rats Jörgen Åstrand*1 and Per Aspenberg1,2
}

Address: ${ }^{1}$ Department of Orthopaedics, Lund University Hospital, Lund, Sweden and ${ }^{2}$ Division of Orthopaedics, Department of Neuroscience and Locomotion, Faculty of Medicine, Linköping University, Linköping, Sweden

E-mail: Jörgen Åstrand* - jorgen.astrand@ort.lu.se; Per Aspenberg - Per.Aspenberg@inr.liu.se

*Corresponding author

This article is available from: http://www.biomedcentral.com/I47I-2474/3/19

(C) 2002 Åstrand and Aspenberg; licensee BioMed Central Ltd. This article is published in Open Access: verbatim copying and redistribution of this article are permitted in all media for any non-commercial purpose, provided this notice is preserved along with the article's original URL.

\begin{abstract}
Background: Avascular necrosis of bone (osteonecrosis) can cause structural failure and subsequent deformation, leading to joint dysfunction and pain. Structural failure is the result of resorption of necrotic bone during revascularization, before new bone has formed or consolidated enough for loadbearing. Bone resorption can be reduced by bisphosphonates. If resorption of the necrotic bone could be reduced during the revascularization phase until sufficient new bone has formed, it would appear that structural failure could be avoided.
\end{abstract}

Methods: To test whether resorption of necrotic bone can be prevented, structural grafts were subjected to new bone ingrowth during systemic bisphosphonate treatment in a rat model.

Results: In rats treated with alendronate the necrotic bone was not resorbed, whereas it was almost entirely resorbed in the controls.

Conclusion: Systemic alendronate treatment prevents resorption of necrotic bone during revascularization. In patients with osteonecrosis, bisphosphonates may therefore prevent collapse of the necrotic bone.

\section{Background}

Osteonecrosis is caused by insufficient circulation. This situation can occur after trauma or be the result of other events or conditions that can compromise circulation, such as corticosteroid treatment, scuba diving, sickle cell anaemia, alcoholism and pregnancy. Osteonecrosis can lead to structural failure, with subsequent collapse and deformation of the bone leading to joint dysfunction and pain. Gardeniers [1] performed a series of experiments, where a section of the femoral head in goats was removed and reattached using bone cement. The cement layer prevented revascularization, which was verified with angiography and histology. No collapse occurred, and mechanical testing of the avascular segment showed no weakening compared to vital controls. However, when a hole was drilled through the cement layer in a second operation, revascularization ocurred and collapse of the femoral head segment followed. Mechanical testing confirmed weakening of the revascularized segments.

Others have confirmed that necrotic bone retains load bearing capacity [2], and evidence can also be found in tools made of bone, which have been known since neolithicum. Consequently the death of bone cells per se does not cause structural failure. Rather, it is caused by the resorption of necrotic bone [3][1]. Resorption is mediated 


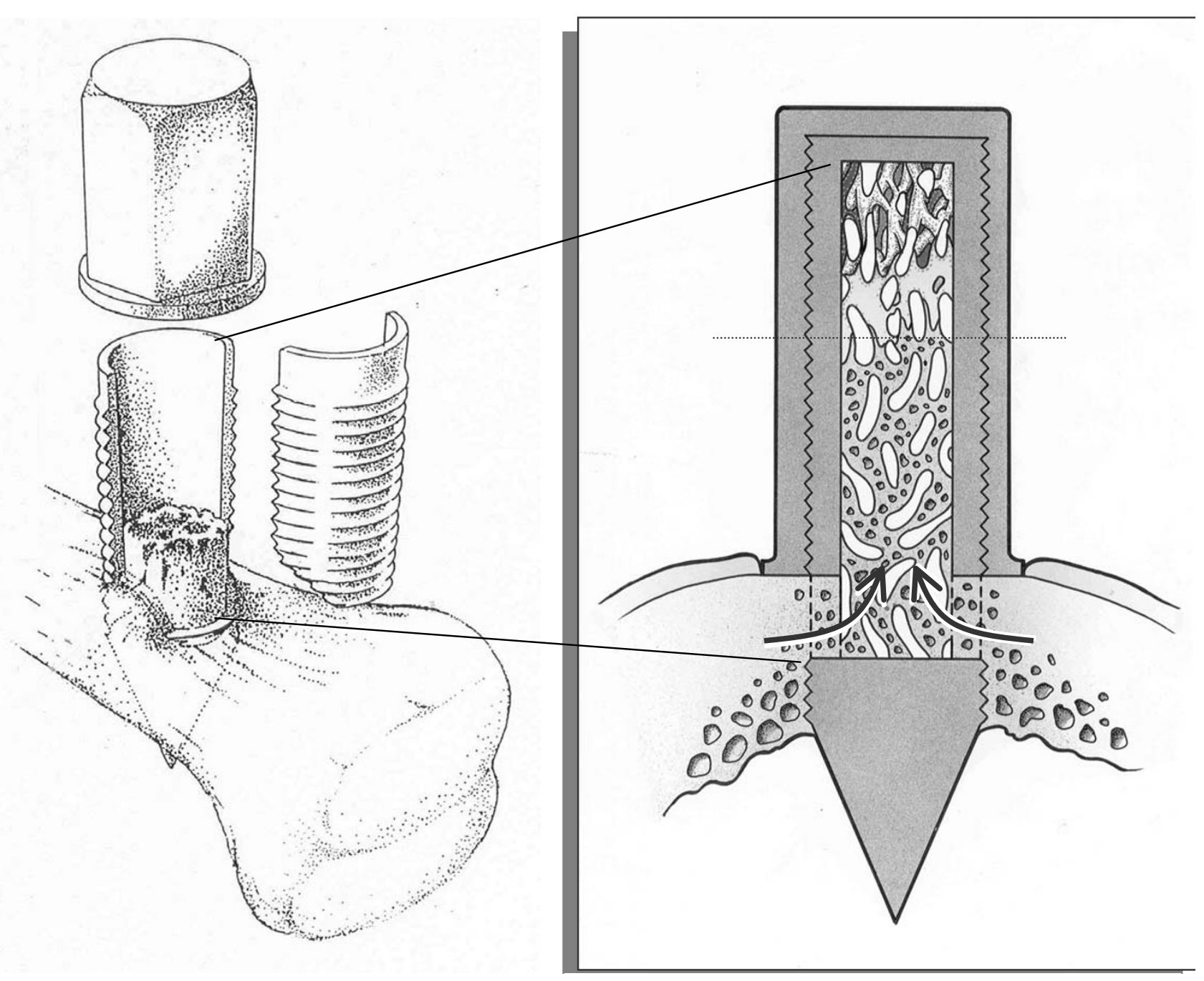

Figure I

A schematic drawing of the Bone Conduction Chamber (BCC). Frozen bone allografts are placed for 6 weeks in the chambers, which are implanted in the proximal tibia of rats. The openings at the lower end of the chamber are placed just below the periosteum. Tissue grows into these openings (arrows) and penetrates the whole of the graft. Ossification reaches about halfway from the bottom towards the top (dashed line). From Aspenberg P, Wang J-S. Eur J Exp Musculoskel Res. 1993;2:69-74. Published with permission from Taylor \& Francis.

by osteoclasts, which are of hematopoetic origin [4] and occurs during or following the revascularization of the necrotic tissue [3][1]. Together with the resorption there is a healing response, with new bone formation within the revascularized tissue.

If the bone resorption associated with osteonecrosis can be inhibited or delayed until sufficient new bone has formed, it would appear that structural failure and its consequences could be avoided. Osteoclastic activity can be reduced with bisphosphonates, a class of drugs in clinical use for the treatment of osteoporosis, Paget's disease and osteolytic metastases. Bisphosphonates bind to bone min- eral and when bone is resorbed by osteoclasts, bisphosphonates are internalized and interfere with cell metabolism which leads to apoptosis. Thus, the osteoclasts are targeted by the treatment.

We used a previously described rat model [5] to study whether bisphosphonate treatment could reduce or inhibit the resorption of necrotic bone. During systemic treatment with bisphosphonate or saline, bone was allowed to grow into necrotic bone grafts inside a bone chamber. This model was chosen in order to standardize the composition, age, size and geometry of the specimens for analysis. 


\section{Methods}

We used the bone conduction chamber (BCC) [5]. The chamber consists of a titanium screw with a cylindrical interior space. It is made up of two threaded half cylinders held together by a hexagonal closed screw cap. One end of the implant is screwed into the bone. The interior of the chamber has a diameter of $2 \mathrm{~mm}$, and is $7 \mathrm{~mm}$ long. There are two bone ingrowth openings located at the bone end of the chamber (Figure 1). Thus, the ingrowing tissues enter the cylindrical space from the bone compartment. The chamber space extends far out into the subcutaneous region and the ingrown bone-derived tissue can proliferate in the chamber without competition with other tissues. Ingrowing tissue, most of it bone, will fill a portion of the chamber within 6 weeks but will not reach the far end of the cylinder. Thus, the tissue ingrowth distance from the holes towards the other end of the chamber can be measured as an estimate for tissue regeneration. Due to the geometry of the chamber it is easy to define areas for histomorphometry. Placing an osteoconductive material, such as a bone graft, in the chamber can increase the tissue ingrowth distance [6].

Pairs of structurally intact cancellous bone grafts were obtained from 18 female Sprague Dawley rats $(200 \mathrm{~g})$ as follows. A cylindrical $2 \times 6 \mathrm{~mm}$ bone rod was resected in the axial direction from the knee joint with a hole cutter. The epiphysis and the growth plate were excised. The proximal part of the graft had the most dense cancellous bone and was later turned towards the ingrowth end of the chamber. The grafts were kept sterile and frozen at $-70^{\circ} \mathrm{C}$.

An alendronate solution was prepared by dissolvling one tablet of alendronate $10 \mathrm{mg}$ (Fosamax, MSD, Malmö, Sweden) in $10 \mathrm{ml}$ water for 1 hour under stirring and then filtering through a sterile Millipore filter, pore size $0.2 \mu \mathrm{m}$. This stock solution was then diluted in saline to the desired concentrations.

After thawing, the grafts were placed in bone chambers and implanted unilaterally in 34 recipient $350 \mathrm{~g}$ male Sprague Dawley rats. Subcutaneous $0.5 \mathrm{ml}$ injections were given postoperatively three days a week. A high dose group was given alendronate at a dose of $205 \mu \mathrm{g} \times \mathrm{kg}^{-1} \times$ day $^{-1}(\mathrm{n}=10)$ with a control group given saline $(\mathrm{n}=10)$. A low dose group was given $4 \mu \mathrm{g} \times \mathrm{kg}^{-1} \times$ day-1 $^{-1}(\mathrm{n}=8)$, with separate controls $(\mathrm{n}=7)$ given saline. The animals for the both dose group and their donors $(n=30)$ were obtained from M \& B (M \& B A/S, P.O. box 1079, DK8680, Ry, Denmark). After 6 weeks, the rats were killed and the contents of the chambers prepared for decalcified histology with sections parallel to the long axis of the chamber, stained with hematoxyllin and eosin.
To monitor the systemic effect of alendronate, $6 \mathrm{~mm}$ of the proximal, unoperated tibial metaphyses was cut at a right angle to the long axis of the bone and weighed before and after ashing at $1000^{\circ} \mathrm{C}$ for 24 hours in all rats.

\section{Evaluation}

For each specimen, 3 sections, each $0.3 \mathrm{~mm}$ apart, were studied. All sections were blinded for identity and evaluated in random order. The evaluation was done by manual point counting of an area of interest ranging from the bottom of the chamber (at the ingrowth end) to the frontier of the advancing new bone formation. The area of interest comprised the central third of the specimen, so that the bone close to the titanium side walls was not included. The total number of points, points covering bone in general and points covering new living bone were counted. The distinction between graft bone and new bone was based on matrix staining (which is paler and more uneven in the graft), presence of osteocytes and trabecular shape. On average 366 points were counted per specimen. In a previous similar experiment, the same person repeated the point counting after one year. The measurement error (sd) was found to be 6 percent units for graft bone, 6 percent units for for new bone, and 3 percent units for bone in general [7]. The bone ingrowth distance was measured with computer-aided methods as described previously [6]. For all measurements, data from the 3 segments were averaged to form one value per graft. Statistical analysis was then done with Mann-Whitney U-test. Institutional guidelines for the treatment and care of experimental animals were followed.

\section{Results}

One animal was lost before harvest due to postoperative complications. In all the others, the histological analysis showed that soft tissue had invaded the whole grafts. New bone had formed a bone ingrowth frontier halfway through the graft. In the controls the grafts appeared resorbed behind this frontier, so that a large marrow cavity had formed behind the frontier, with haematogenous bone marrow (Fig 2A). In contrast, the grafts in rats treated with the high alendronate dose remained intact, and new bone lined the graft trabeculae, leaving only little space for haematogenous marrow (Fig 2B). The difference in bone density was clearly visible to the naked eye. Grafts in rats treated with the low alendronate dose were more similar in appearance to the controls with resorption of the graft and marrow cavity formation but also some areas with denser bone comprising both graft bone and newly formed bone.

In specimens treated with the high dose alendronate, there was no difference in the distance advanced by the bone ingrowth frontier compared to controls (alendronate $2.8 \mathrm{~mm}$. sd 1.1, control $2.5 \mathrm{~mm}$. sd 1.0). Of the space 
A
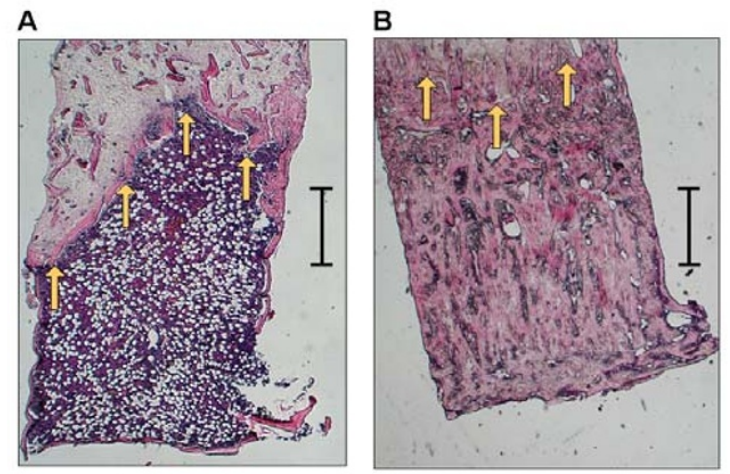

\section{Figure 2}

(A) Section of a graft in a control specimen. Bone ingrowth has ocurred from the lower end of the picture and upwards. The bone formation frontier has reached roughly halfway through the graft (arrows). Behind the frontier a marrow cavity has formed, and inside the cavity the graft is almost entirely resorbed. (B) Section of a graft in a rat treated with the high dose of alendronate. The ossification has reached the same distance (arrows), but the graft is not resorbed. Hematoxylin Eosin, bar lengths I mm.

behind the bone ingrowth frontier, 70\% (sd 18) consisted of graft or host bone, whereas in the controls there was $27 \%$ (sd 13) ( $p=0.0002)$. About half of the bone in the alendronate treated specimens was new living bone that had formed upon the surfaces of the remaining graft trabecula. Thus, host bone comprised 37\% (sd 15) of the total volume. In the controls it was $19 \%$ ( sd 9) $(\mathrm{p}=0.005$; table 1).

In rats treated with the low dose of alendronate, graft and host bone content was 29\% (sd 11) and in the controls $18 \%$ (sd 9, p = 0.049). Host bone comprised 24\% (sd 9) of the total volume and in the controls 15\% (sd 8) (n.s.). Again, there was no difference in the bone ingrowth distance (alendronate $2.11 \mathrm{~mm}$ sd 0.5 , controls $2.07 \mathrm{~mm}$ sd $0.5)$.

In a previous study the bone content of unimplanted grafts was $38 \%$ (sd 10) [7]. This is similar to the remaining graft content of the high dose alendronate treated specimens, which was $34 \%$ (sd 5), with controls containing $8 \%$ (sd 4$)(\mathrm{p}=0.0002)$. In the low dose alendronate specimens less of the grafts remained, $5 \%$ (sd 2), and in the controls 3\% (sd 3) $(\mathrm{p}=0.037)$. (Table 1)

All alendronate treated rats had higher ashweight than their controls. This difference was more pronounced in the high dose group (alendronate treated $136.3 \mathrm{mg}$ s.d. 10 , controls $57.3 \mathrm{mg}$ s.d. $3, \mathrm{p}=0.0002$ ) than in the low dose group (alendronate treated $86.7 \mathrm{mg}$ s.d. 9, controls $60.6 \mathrm{mg}$ s.d. $4, \mathrm{p}=0.0012$ ).

\section{Discussion}

Structural failure associated with osteonecrosis is caused by resorption of the necrotic bone [3], [1]. If bone resorption could be avoided during revascularization and formation of new bone, structural failure and collapse could possibly also be avoided. In an uncontrolled clinical study, Agarwala et al treated 16 patients with avascular necrosis of the hip with alendronate and found improvement in pain, disability and function compared to the expected natural history of the disease, and MRI remained stable [8]. Although the results are interesting, the lack of a control group limits the conclusions possible to draw from that study and the authors states that the beneficial action of alendronate in avascular necrosis of the hip is not clear. It could be hypothesised that alendronate prevented structural failure, deformation and subsequently pain. In the present study, histological evaluation showed that resorption of a necrotic bone graft during revascularisation and bone ingrowth was prevented with systemic bisphosphonate treatment.

Allografts were used as a model for necrotic, autologous bone. The strain of Sprague-Dawley rats used for the high dose group is, however, inbred to such an extent that no differences can be detected in the incorporation of autoversus allografts in this model [9]. Theoretically, it could be assumed that also after freezing and thawing, allografts are more immunogenic than autologous bone and more prone to resorption. Since we wanted to study whether it is possible to reduce bone resorption during revascularization, such an increased tendency to resorption would necessitate an even greater protective effect of alendronate in this model.

This study was performed in two phases. First, we tested the principal possibility, by using a high alendronate dose. When this experiment yielded a positive response, we tested whether also a lower dose, at the level of osteoporosis treatment, could be effective.

In humans, the oral alendronate dose recommended for use against osteoporosis is $10 \mathrm{mg}$ daily. Due to the low gastrointestinal uptake of about $0.6 \%$, this would correspond to an injection of $1 \mu \mathrm{g} \times \mathrm{kg}^{-1} \times \mathrm{day}^{-1}$. In this study, the low dose group received $4 \mu \mathrm{g}$ alendronate $\times \mathrm{kg}^{-1} \times$ day $^{-}$ 1 , a difference corresponding to the difference in metabolic rate. The alendronate dose in the high dose group was 50 times higher. Both the bisphosphonate treated groups in this study showed less bone resorption than their respective controls, measurable as increased total bone con- 
Table I:

\begin{tabular}{llll}
\hline & total bone volume & host bone volume & graft bone volume \\
\hline & & & $34 \%(s d 5)$ \\
alendr, high dose & $70 \%(s d ~ 18)$ & $37 \%(s d ~ I 5)$ & $8 \%(s d 4)$ \\
contr, high dose & $27 \%(s d ~ I 3)$ & $19 \%(s d 9)$ & $(p=0.0002)$ \\
& $(p=0.0002)$ & $(p=0.005)$ & $5 \%(s d 2)$ \\
alendr, low dose & $29 \%(s d ~ I I)$ & $24 \%(s d 9)$ & $3 \%(s d 3)$ \\
contr, low dose & $18 \%(s d 9)$ & $15 \%(s d 8)$ & $(p=037)$ \\
not implanted grafts (ref [7]) & $(p=0.049)$ & n.s. & $38 \%(s d ~ 10)$ \\
\hline
\end{tabular}

tent and graft bone content. However, in the low dose group this difference was less pronounced.

We also found a significant increase in host bone formation by alendronate in the high dose group. In a previous chamber study, in which grafts were pre-treated with topical alendronate, we also saw this effect: new bone formation was increased in alendronate treated specimens [7]. Bone ingrowth into the chambers is enhanced by the presence of a structural bone graft [6]. Thus, the increased bone formation could be explained by a "scaffolding" effect: if the graft is preserved, as in the treated groups, there are more bone graft surfaces on which new bone formation can occur.

Another explanation for the increase in new bone formation seen in alendronate treated groups would be a direct effect of alendronate stimulating new bone formation. There are reports of osteoblastic stimulation by bisphosphonates in vitro [10] and in vivo effects upon osteoblasts have been suggested [11].

\section{Conclusions}

In conclusion, systemic bisphosphonate treatment can reduce the resorption of necrotic bone. This could prove useful in clinical situations, for example as a treatment to reduce the risk of structural failure and collapse after osteonecrosis. Although high dose parenteral treatment may become necessary for full effect, also lower perorally administered doses appeared to be of some value.

\section{Competing interests}

None declared.

\section{Acknowledgements}

The authors thank Inger Mårtensson and Carina Forslund for technical assistance and Mats Christensen for manufacturing the implants.

\section{References}

I. Gardeniers JWM: Behaviour of normal, avascular and revascularizing cancellous bone. Nijmegen: Catholic University of Nijmegen; Monography. 1988
2. Parks NL, Engh GA: The Ranawat Award. Histology of nine structural bone grafts used in total knee arthroplasty. Clin Orthop 1997, 17-23

3. Glimcher MJ, Kenzora JE: The biology of osteonecrosis of the human femoral head and its clinical implications. III. Discussion of the etiology and genesis of the pathological sequelae; commments on treatment. Clin Orthop 1979, 273-312

4. Quinn JM, Sabokbar A, Athanasou NA: Cells of the mononuclear phagocyte series differentiate into osteoclastic lacunar bone resorbing cells. J Pathol 1996, 179:106-III

5. Wang JS, Aspenberg P: Basic fibroblast growth factor and bone induction in rats. Acta Orthop Scand 1993, 64:557-56 I

6. Wang JS, Aspenberg P: Basic fibroblast growth factor promotes bone ingrowth in porous hydroxyapatite. Clin Orthop 1996, 252260

7. Aspenberg P, Astrand J: Bone Allografts Pretreated with a Bisphosphonate Do Not Resorb. Acta Orthop Scand 2002, 73:20-23

8. Agarwala S, Sule A, Pai BU, Joshi VR: Alendronate in the treatment of avascular necrosis of the hip. Rheumatology (Oxford) 2002, $41: 346-347$

9. Thoren K, Aspenberg P: Increased bone ingrowth distance into lipid-extracted bank bone at 6 weeks. A titanium chamber study in allogeneic and syngeneic rats. Arch Orthop Trauma Surg 1995, I| 4:167-|7|

10. Reinholz GG, Getz B, Pederson L, Sanders ES, Subramaniam M, Ingle JN, Spelsberg TC: Bisphosphonates directly regulate cell proliferation, differentiation, and gene expression in human osteoblasts. Cancer Res 2000, 60:600I-6007

II. Bobyn D, Hacking A, Tanzer M, Richards C, Harvey E, Little D: Marked enhancment of bone growth into porous implants by zoledronic acid. In: 48th Annual Meeting of the Orthopaedic Research Society 2002, paper 0027

\section{Pre-publication history}

The pre-publication history for this paper can be accessed here:

http://www.biomedcentral.com/1471-2474/3/19/prepub 